DAMTP-2004-87 MIFP-04-17

hep-th/0408217

\title{
The First Law of Thermodynamics for Kerr-Anti-de Sitter Black Holes
}

\author{
G.W. Gibbons $\sharp$, M.J. Perry ${ }^{\sharp}$ and C.N. Pope ${ }^{\ddagger}$ \\ ${ }^{\sharp}$ DAMTP, Centre for Mathematical Sciences, Cambridge University \\ Wilberforce Road, Cambridge CB3 OWA, UK \\ $\ddagger$ George P. E Cynthia W. Mitchell Institute for Fundamental Physics, \\ Texas AEM University, College Station, TX 77843-4242, USA
}

\begin{abstract}
$\underline{\text { ABSTRACT }}$
We obtain expressions for the mass and angular momenta of rotating black holes in anti-de Sitter backgrounds in four, five and higher dimensions. We verify explicitly that our expressions satisfy the first law of thermodynamics, thus allowing an unambiguous identification of the entropy of these black holes with $\frac{1}{4}$ of the area. We find that the associated thermodynamic potential equals the background-subtracted Euclidean action multiplied by the temperature. Our expressions differ from many given in the literature. We find that in more than four dimensions, only our expressions satisfy the first law of thermodynamics. Moreover, in all dimensions we show that our expression for the mass coincides with that given by the conformal conserved charge introduced by Ashtekar, Magnon and Das. We indicate the relevance of these results to the AdS/CFT correspondence.
\end{abstract}

\footnotetext{
$\ddagger$ Research supported in part by DOE grant DE-FG03-95ER40917.
} 


\section{Contents}

1 Introduction 2

2 Kerr-AdS Black Holes in Four Dimensions $\quad 7$

2.1 Thermodynamics in four-dimensional Kerr-AdS . . . . . . . . . . . . 7

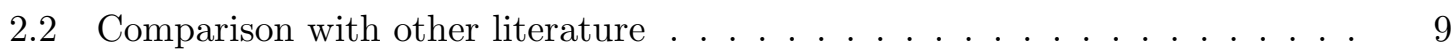

3 Kerr-AdS Black Holes in Five Dimensions 11

3.1 Thermodynamics in five-dimensional Kerr-AdS . . . . . . . . . . . . . . . 11

3.2 Comparison with other literature . . . . . . . . . . . . . . . 13

3.3 Conformal infinity in the Hawking-Hunter-Taylor-Robinson metrics . . . . . 16

4 Kerr-AdS Black Holes in $D \geq 6$ Dimensions $\quad 18$

4.1 Thermodynamics in $D \geq 6$ Kerr-AdS . . . . . . . . . . . . . . . 18

4.2 Comparison with other literature . . . . . . . . . . . . . . . 21

5 Thermodynamic Energy and the Conformal Mass 22

6 Conclusions 24

$\begin{array}{ll}\text { A Euclidean Action of the Kerr-AdS Metrics } & 27\end{array}$ 


\section{Introduction}

Although of no great direct astrophysical or cosmological importance, there has been considerable interest of late in the properties of black holes in higher dimensions and in backgrounds with a negative cosmological constant. The main reason for this interest has been the conjectured AdS/CFT correspondence, which allows statements to be made about fourdimensional quantum field theory using solutions of the Einstein equations with a negative cosmological constant in five dimensions. In particular the thermodynamic properties of five-dimensional rotating black holes in an anti-de Sitter background give information about four-dimensional quantum field theory at non-zero temperature in a state of rigid rotation. There are obvious extensions to higher and lower dimensions, and to charged black holes. The requisite five-dimensional solution, and some specialised higher-dimensional solutions, have been available for some time [1]. In recent work [2], the most general uncharged Kerrde Sitter solutions in arbitrary dimension $D$ have been constructed, and also some charged solutions in $D=5[3,4]$.

An examination of the fairly extensive literature reveals that while everyone seems agree as to the area $A$, the temperature $T=\kappa /(2 \pi)$ and the angular velocities $\Omega_{i}$ relative to a non-rotating frame at infinity, there is a lack of unanimity about what precisely are the correct expressions for the total mass or energy $E$, and the total angular momenta $J_{i}$, of the Kerr-anti-de Sitter black holes. Even more worrying, the proffered answers do not always satisfy the first, and hence the second, law of thermodynamics. The principal purpose of the present paper is to rectify this situation. To recall the words of Eddington [5], much quoted during the first gravitational thermodynamic revolution,

The law that entropy always increases - the second law of thermodynamics holds, I think, a supreme position among the laws of Nature. If someone points out to you that your pet theory of the universe is in disagreement with Maxwell's equations, then so much the worse for Maxwell's equations. If it is found to be contradicted by observations - well, these experimentalists do bungle sometimes. But if your theory is found to be against the second law of thermodynamics I can give you no hope; there is nothing for it but to collapse in humiliation.

Perhaps the most elementary approach is to follow the original path of Christodoulou and Ruffini $[6,7]$. That is, to note that a particle of 4 -momentum $p_{\mu}$ will have a conserved energy $^{1} e=-p_{\mu} K^{\mu}$ and conserved angular momentum $j=p_{\mu} m^{\mu}$, where $K^{\mu}$ is the Killing

\footnotetext{
${ }^{1}$ We use the mainly plus signature convention.
} 
vector, timelike near infinity, associated to a non-rotating frame at infinity, and $m^{\mu}$ is the angular Killing field, with closed orbit normalised to have parameter length $2 \pi$. The future directed null generator $l^{\mu}$ of the horizon of the black hole is given by

$$
l^{\mu}=K^{\mu}+\Omega m^{\mu} .
$$

If the particle crosses the event horizon, since the momentum is future directed timelike or null, we shall have

$$
l^{\mu} p_{\mu} \leq 0
$$

or in other words,

$$
-e+\Omega j \leq 0
$$

Now energy and angular momentum of the particle presumably increase the mass or total energy $^{2} E$ by $d E=e$ and the angular momenta by $d J=j$, whence

$$
d E-\Omega d J \geq 0
$$

Christodoulou and Ruffini went further; they noticed that the left-hand side of (1.4) is proportional to an exact differential, and they were led to introduce an irreducible mass $M_{\text {irr }}$ and to express $E$ as a function of $M_{\text {irr }}$ and $J_{i}$. For example, in the case of the fourdimensional Kerr solution (with vanishing cosmological constant), they found what we shall call the Christodoulou-Ruffini Mass Formula

$$
E=\sqrt{M_{\mathrm{irr}}^{2}+\frac{J^{2}}{4 M_{\mathrm{irr}}^{2}}} .
$$

Christodoulou and Ruffini regarded their mass formula as analogous to the special relativistic relation for the kinetic energy $\left(E-M_{\mathrm{irr}}\right)$ of a particle of rest mass $M_{\mathrm{irr}}$,

$$
E=\sqrt{M_{\mathrm{irr}}^{2}+\mathbf{p}^{2}}
$$

Subsequent work revealed that

$$
M_{\mathrm{irr}}=\sqrt{\frac{A}{16 \pi}},
$$

where $A$ is the area of the event horizon, and that (1.4) may be written as

$$
d E-\Omega_{i} d J_{i}=\frac{\kappa}{8 \pi} d A \geq 0 .
$$

\footnotetext{
${ }^{2}$ Here we are using the notation $d E$ in the usual sense of thermodynamics.
} 
This, and Hawking's area-increase law, led to the idea that some multiple of $A$ should play the role of entropy $S$. The multiple, $\frac{1}{4}$, was subsequently fixed by Hawking's discovery of the quantum radiance of black holes. The Christodoulou-Ruffini mass formula could then be recognised as giving the shape of the Gibbs surface in the three-dimensional affine space with coordinates $(S, E, J)$.

Mathematically, the 1-form

$$
d E-T d S-\Omega d J
$$

can be shown to define a contact structure on the five-dimensional space with affine coordinates $(E, T, S, \Omega, J)$, and the Gibbs surface is the projection down to the three-dimensional space spanned by $(E, T, S)$ of a Legendrian submanifold; i.e. the analogue for contact geometry of a Lagrangian submanifold of a symplectic manifold. Indeed, suppressing the $E$ coordinate gives a symplectic four-dimensional space with coordinates $(T, S, \Omega, J)$ and symplectic form

$$
d T \wedge d S+d \Omega \wedge d J .
$$

The two-dimensional surface in four-dimensional space given, say, by expressing $T$ and $\Omega$ as functions of $S$ and $J$ is a Lagrangian submanifold. If the energy is obtained by minimizing the total energy of a system with fixed entropy $S$ and angular momentum $J$, then, as we shall remind the reader later in detail, it is also a mathematical triviality that the first law must hold, which perhaps explains the forcefulness with which Eddington expressed himself.

All these relationships were originally derived by using the particular form of the Kerr solution. However, a general derivation for asymptotically flat solutions with $\Lambda=0$ was then given, by first giving a general proof, using Komar integrals, of another formula which had previously been obtained directly from the Kerr metric functions, namely

$$
\frac{1}{2} E=\frac{\kappa A}{8 \pi}+\Omega J .
$$

Variation of (1.11) through a family of stationary vacuum solutions yields (1.8), i.e. the first law. In fact rewriting (1.11) as

$$
\frac{1}{2} E=T S+\Omega J
$$

allows us to recognise it as a generalised Gibbs-Duhem relation, arising from the first law by a simple scaling argument based on the elementary fact of dimensional analysis that in four spacetime dimensions, $E$ must be a homogeneous function, of degree $\frac{1}{2}$, in $A$ and $J$.

We can also interpret the Smarr-Gibbs-Duhem relation as telling us that the thermodynamic potential

$$
\Phi=\Phi(T, \Omega)=E-T S-\Omega J
$$


is given simply by

$$
\Phi=\frac{1}{2} E
$$

The Smarr-Gibbs-Duhem relation $(1.14)^{3}$, if it holds, is sometimes useful in the Euclidean approach to quantum gravity, because in the statistical mechanics of a Gibbs ensemble of fixed temperature and angular velocity, the partition function satisfies

$$
Z(T, \Omega)=e^{-\beta \Phi}
$$

and therefore thermodynamic potential $\Phi$ is expected to be related to the Euclidean action I quite generally by the Quantum Statistical Relation ${ }^{4}$

$$
\beta \Phi=I
$$

i.e. $Z \equiv e^{-\beta \Phi}=e^{-I}$. The action $I(\beta, \Omega)$ is the Legendre transform of the entropy $S=$ $S(M, J)$, and is referred to in thermodyamics as the Massieu function, often denoted by the symbol $\Gamma$.

If the Smarr relation holds then

$$
I=\frac{1}{2} E \beta
$$

Note that if we have some way of calculating the thermodynamic potential $\Phi$, say by a Euclidean path integral, then we can calculate, or indeed define, the entropy $S$ and angular velocities $J$, via

$$
S=-\frac{\partial \Phi}{\partial T}, \quad J=-\frac{\partial \Phi}{\partial \Omega}
$$

The first law now follows trivially by a Legendre transformation, the mass $E$ being defined by

$$
E=\Phi+T S+\Omega J
$$

Of course the quantities $E$ and $J$ so obtained need have no direct relation to those defined using, say, the ADM mass and angular momentum. In the case of asymptotically-flat black

\footnotetext{
${ }^{3}$ In the elementary thermodynamics of a single-component homogeneous gas, the energy is a homogeneous function of degree of the entropy and volume, and the Gibbs-Duhem relation says that the Gibbs free energy $G=E-T S+P V$ vanishes.

${ }^{4}$ Note that the Quantum Statistical Relation necessarily contains Planck's constant and is quantummechanical in origin. It cannot be deduced from classical thermodynamics and is logically independent of classical thermodynamics. For this reason it seems inappropriate to refer to (1.16) as the Gibbs-Duhem relation, as is done by some authors, since Gibbs died in 1903 and Duhem in 1916, before the advent of quantum mechanics. The adoption of (1.16) for quantum gravity was first proposed in [8].
} 
holes it is well known that at the semi-classical level these two methods agree. If one recalls that

$$
S=\beta \frac{\partial I}{\partial \beta}-I
$$

one realises that the perhaps puzzling possibility, that a classical Euclidean solution of the equations of motion can have a positive entropy, depends on the Euclidean action I's not being proportional to the imaginary time period $\beta$, as it would for an ordinary static soliton for example, but instead having a steeper dependence on $\beta$.

Despite these successes in the asymptotically-flat case, it is important to recognise that there are obvious difficulties in deriving the Smarr formula, and its variation, if the cosmological constant is negative. Various infinite integrals are encountered, and even the evaluation of the Komar integrals requires care [9]. Moreover, there is no longer a scaling symmetry, although it may be re-instated to some extent by rescaling the cosmological constant. Thus, while the extension of the above ideas to asymptotically flat vacuum rotating black holes in higher dimensions follows in a straightforward fashion, once the solutions are known, the extension to asymptotically anti-de Sitter rotating black holes is, as we shall see, not so simple.

The plan of the paper is as follows. In section 2, we treat four-dimensional KerrAdS black holes, and show that with the correct choice of energy and angular momentum, consistent with $S O(3,2)$ invariance, the first law of thermodynamics holds, and the entropy is given by $\frac{1}{4}$ of the area $A$. We also verify that the quantum statistical relation (1.16). The section concludes with a detailed comparison with other expressions given in the literature for both the energy and the angular momentum. We show that these do not satisfy the first law of thermodynamics.

Section 3 is devoted to the five-dimensional case, where we obtain analogous results that are consistent with the first law of thermodynamics. Again we give a comparison with various expressions suggested in the literature, showing that they fail to satisfy the first law. Section 6 contains a description of the two different approaches to the conformal boundary at infinity, which seem to be responsible for the differences between the results in the literature.

Section 4 generalises these results to all dimensions $D \geq 6$. We give results for the mass, angular momenta and actions for the general Kerr-AdS metrics in all dimensions. Some details of the calculation of the action are relegated to Appendix A.

In section 5, we show that in all dimensions, our expression for the mass of the general Kerr-AdS black hole coincides with that given by the conformal conserved charge introduced 
by Ashtekar, Magnon and Das [13,25].

Finally, section 6 contains our conclusions.

\section{Kerr-AdS Black Holes in Four Dimensions}

\subsection{Thermodynamics in four-dimensional Kerr-AdS}

The four-dimensional Kerr-(anti)-de Sitter metric was obtained by Carter [10]. It can be written as

$$
d s_{4}^{2}=-\frac{\Delta}{\rho^{2}}\left[d t-\frac{a}{\Xi} \sin ^{2} \theta d \phi\right]^{2}+\frac{\rho^{2} d r^{2}}{\Delta}+\frac{\rho^{2} d \theta^{2}}{\Delta_{\theta}}+\frac{\Delta_{\theta} \sin ^{2} \theta}{\rho^{2}}\left[a d t-\frac{r^{2}+a^{2}}{\Xi} d \phi\right]^{2},
$$

where

$$
\begin{aligned}
\Delta & \equiv\left(r^{2}+a^{2}\right)\left(1+r^{2} l^{-2}\right)-2 m r, \quad \Delta_{\theta} \equiv 1-a^{2} l^{-2} \cos ^{2} \theta, \\
\rho^{2} & \equiv r^{2}+a^{2} \cos ^{2} \theta, \quad \Xi \equiv 1-a^{2} l^{-2},
\end{aligned}
$$

and it satisfies $R_{\mu \nu}=-3 l^{-2} g_{\mu \nu}$.

The outer horizon is located at the radius $r=r_{+}$, where $r_{+}$is the largest root of $\Delta=0$. The area of the event horizon is

$$
A=\frac{4 \pi\left(r_{+}^{2}+a^{2}\right)}{\Xi}
$$

The surface gravity $\kappa$ and inverse temperature $\beta$ are given by

$$
\beta=T^{-1}=\frac{2 \pi}{\kappa}=\frac{4 \pi\left(r_{+}^{2}+a^{2}\right)}{r_{+}\left(1+a^{2} l^{-2}+3 r_{+}^{2} l^{-2}-a^{2} r_{+}^{-2}\right)} .
$$

The angular velocity of the black hole, measured relative to a frame that is non-rotating at infinity, is given by

$$
\Omega=\frac{a\left(1+r_{+}^{2} l^{-2}\right)}{r_{+}^{2}+a^{2}} .
$$

Note that Hawking, Hunter and Taylor-Robinson define an angular velocity which is measured relative to a frame rotating at infinity, by

$$
\Omega^{\prime}=\frac{a \Xi}{r_{+}^{2}+a^{2}}
$$

and so

$$
\Omega-\Omega^{\prime}=\frac{a}{l^{2}} .
$$


The action $I_{4}$ of the four-dimensional Kerr-AdS black hole turns out to be given by

$$
\begin{aligned}
I_{4} & =\frac{\beta}{2 \Xi}\left[m-r_{+} l^{-2}\left(r_{+}^{2}+a^{2}\right)\right], \\
& =-\frac{\pi\left(r_{+}^{2}+a^{2}\right)^{2}\left(r_{+}^{2} l^{-2}-1\right)}{\Xi l^{2}\left(3 r_{+}^{4} l^{-2}+\left(1+a^{2} l^{-2}\right) r_{+}^{2}-a^{2}\right)} .
\end{aligned}
$$

(See Appendix A for a discussion of the calculation of the action of the Kerr-AdS metrics in arbitrary dimension.)

The mass and angular momentum are related to the parameters $m$ and $a$ appearing in the metric; they were first obtained by Henneaux and Teitelboim [11]. Their approach was a Hamiltonian one, and they obtained the generators of $S O(3,2)$, i.e. in modern language the values of the moment maps for the action of $S O(3,2)$ on the gravitational phase space, and checked their Poisson brackets. For these solutions the Abbot-Deser masses [12] and the Ashtekar-Magnon [13] masses give the same answers. The Komar technique of Magnon [9] also gives the same answers for angular momentum and, with care, for the mass. Henneaux and Teitelboim find the dimensionless $S O(3,2)$ generators to be

$$
J_{51}=\frac{16 \pi m l}{\Xi^{2}}, \quad J_{23}=-\frac{16 \pi m a}{\Xi^{2}} .
$$

The above considerations lead naturally to the following expressions for the "physical" mass (or energy) $E$ and angular momentum $J$ :

$$
E=\frac{m}{\Xi^{2}}, \quad J=\frac{m a}{\Xi^{2}}
$$

We shall discuss different choices that can be found in the literature later. For now, we note that Kostelecky and Perry [14] obtained the generators for Kerr-Newman black holes, and that their expressions agree with those of Henneaux and Teitelboim in the uncharged case. It is also worth remarking that the calculation of $J$, either via the Komar integral or other approaches, is relatively straightforward and unambiguous. By contrast, the calculation of $E$ is trickier; for example, one encounters a divergence when performing a Komar integral, and this leads to ambiguities in performing a subtraction to obtain a finite result.

It is easily verified that with the definitions (2.12) of mass and angular momentum, and with the angular velocity $\Omega$ relative to a non-rotating frame at infinity given by (2.6), then the first law of thermodynamics holds, namely

$$
d E=T d S+\Omega d J
$$

with the entropy

$$
S=\frac{1}{4} A
$$


Note that our identification of the entropy $S$ with $\frac{1}{4}$ of the area $A$ depends crucially upon the fact that the first law of thermodynamics holds; in particular, the right-hand side of (2.13) is exact if and only if the coefficient is $\frac{1}{4}$. Without this, we could merely give a hand-waving argument based on the idea that both $S$ and $A$ are non-decreasing functions.

It is also easily verified that these quantities satisfy the Quantum Statistical Relation

$$
E-T S-\Omega J=T I_{4}
$$

It is useful to note that one can reverse the logic, and calculate the energy $E$ (up to an additive constant) by integration of (2.13). Since, as we remarked above, the expression for $J$ is on "firmer ground" than that for $E$, and of course the expressions for $T, S$ and $\Omega$ are uncontroversial, this can provide a useful way of checking the validity of a proposal for $E$. It is, of course, essential that the right-hand side of (2.13) be an exact differential if this procedure to determine $E$ is to make sense. With $T, \Omega, J$ and $S$ given as in (2.5), (2.6), (2.12) and (2.14), it is straightforward to verify that the right-hand side of (2.13) is indeed exact, and by integration one can then recover the expression for $E$ given in (2.12). It is natural to choose the constant of integration so that $E$ vanishes when the parameter $m$ vanishes, i.e. for the AdS metric. ${ }^{5}$

\subsection{Comparison with other literature}

In some of the literature, a different expression for the mass or energy of the four-dimensional Kerr-AdS black hole has been given. For example, Hawking, Hunter and Taylor-Robinson give the expression

$$
E^{\prime}=\frac{m}{\Xi}
$$

for the mass, while still taking $J=m a / \Xi^{2}$, as in (2.12), for the angular momentum. They note that with this choice, the "usual thermodynamic relations" imply that the entropy is indeed given by (2.14), as one would expect. By this, they appear to mean that a version of the Quantum Statistical Relation holds, namely

$$
E^{\prime}-T S-\Omega^{\prime} J=T I_{4}
$$

Note that this version differs from the relation (2.15) that is satisfied by the mass $E=m / \Xi^{2}$, since there the angular velocity $\Omega$, given in (2.6), measured relative to a non-rotating frame at infinity was used, whereas in (2.17) it is the angular velocity $\Omega^{\prime}$, given in (2.7), measured

\footnotetext{
${ }^{5}$ In the literature on the Ads/CFT correspondence this is not always done, and in five dimensions, but not in four dimensions, $E$ contains a Casimir contribution. This of course would break $S O(4,2)$ invariance.
} 
relative to a frame rotating at infinity, that must be used. The relation between (2.15) and (2.17) can easily be understood by noting from (2.8), (2.12) and (2.16) that

$$
E^{\prime}-\Omega^{\prime} J=E-\Omega J
$$

What is not commented upon in [1] is the issue of the first law of thermodynamics. It is easily verified that not only is it not satisfied with these definitions, i.e.

$$
d E^{\prime} \neq T d S+\Omega^{\prime} d J
$$

but the right-hand side of (2.19) is not even an exact differential, and thus it cannot be integrated to give any energy function. In other words, at least if one adopts the universallyaccepted expression $J=m a / \Xi^{2}$ for the angular momentum, it is impossible to satisfy the first law of thermodynamics if the angular velocity $\Omega^{\prime}$, defined in (2.7), is used in (2.19). Rather, one should use the angular velocity $\Omega$, which is measured relative to a non-rotating frame at infinity, and which is defined in (2.6). ${ }^{6}$ As we have noted, by integrating the right-hand side of (2.13) one obtains the result $E=m / \Xi^{2}$ that was given in $[11,14]$.

Caldarelli et al. [15] agree with our formulae (2.12), which they obtained using the Brown-York [16] method. Silva [17] agrees with the angular momentum in (2.12) but obtains for the energy the same expression (2.16) as Hawking, Hunter and Taylor-Robinson. He notes without comment that to get the first law to turn out correctly, he must divide his expression for the energy by $\Xi$.

It is interesting to note that in [15] a so-called "Smarr formula" (which we prefer to call a "Christodoulou-Ruffini mass formula") is derived. It is given by

$$
E=\sqrt{M_{\mathrm{irr}}^{2}\left(1+4 M_{\mathrm{irr}}^{2} l^{-2}\right)^{2}+\frac{1}{4} J^{2} M_{\mathrm{irr}}^{-2}\left(1+4 M_{\mathrm{irr}}^{2} l^{-2}\right)} .
$$

The failure of the Smarr-Gibbs-Duhem relation in the presence of the cosmological constant, due to its breaking of scale invariance, may be seen from the fact that

$$
\frac{1}{2} E-T S-\Omega J=\frac{\left(r_{+}^{2}+a^{2}\right)\left(a^{2} r_{+}^{2}-a^{2} l^{2}-2 l^{2} r_{+}^{2}\right)}{4\left(a^{2}-\ell^{2}\right)^{2} r_{+}} \neq 0 .
$$

(See [18] for a discussion of thermodynamics in a system of gravity coupled to non-linear electrodynamics, where the absence of a scaling symmetry also leads to a breakdown of the Smarr formula, but the first law continues to hold.)

It is also shown in [15] that starting from the action $I_{4}$ one may calculate the thermodynamic potential $\Phi(T, \Omega)$, and hence $S$ and $J$, which agree with (2.12). Performing the

\footnotetext{
${ }^{6}$ The importance of measuring the angular velocity relative to a non-rotating frame was emphasised by Caldarelli, Cognola and Klemm [15].
} 
Legendre transform they obtain the mass $E(S, J)$, which agrees with (2.12). And finally, it is stated in [15] that these expressions, once a partial Legendre transform has been made to the Helmholtz free energy

$$
F(T, J)=\Phi+\Omega J
$$

give the correct expressions for the entropy $S$ and the angular velocity $\Omega$.

\section{Kerr-AdS Black Holes in Five Dimensions}

\subsection{Thermodynamics in five-dimensional Kerr-AdS}

The five-dimensional Kerr-(anti)-de Sitter metric was obtained by Hawking, Hunter and Taylor-Robinson [1]. It is given by

$$
\begin{aligned}
d s_{5}^{2}= & -\frac{\Delta}{\rho^{2}}\left[d t-\frac{a \sin ^{2} \theta}{\Xi_{a}} d \phi-\frac{b \cos ^{2} \theta}{\Xi_{b}} d \psi\right]^{2}+\frac{\Delta_{\theta} \sin ^{2} \theta}{\rho^{2}}\left[a d t-\frac{r^{2}+a^{2}}{\Xi_{a}} d \phi\right]^{2} \\
& \frac{\Delta_{\theta} \cos ^{2} \theta}{\rho^{2}}\left[b d t-\frac{r^{2}+b^{2}}{\Xi_{b}} d \psi\right]^{2}+\frac{\rho^{2} d r^{2}}{\Delta}+\frac{\rho^{2} d \theta^{2}}{\Delta_{\theta}} \\
& +\frac{\left(1+r^{2} l^{-2}\right)}{r^{2} \rho^{2}}\left[a b d t-\frac{b\left(r^{2}+a^{2}\right) \sin ^{2} \theta}{\Xi_{a}} d \phi-\frac{a\left(r^{2}+b^{2}\right) \cos ^{2} \theta}{\Xi_{b}} d \psi\right]^{2},
\end{aligned}
$$

where

$$
\begin{aligned}
\Delta & \equiv \frac{1}{r^{2}}\left(r^{2}+a^{2}\right)\left(r^{2}+b^{2}\right)\left(1+r^{2} l^{-2}\right)-2 m, \\
\Delta_{\theta} & \equiv 1-a^{2} l^{-2} \cos ^{2} \theta-b^{2} l^{-2} \sin ^{2} \theta \\
\rho^{2} & \equiv r^{2}+a^{2} \cos ^{2} \theta+b^{2} \sin ^{2} \theta \\
\Xi_{a} & \equiv 1-a^{2} l^{-2}, \quad \Xi_{b} \equiv 1-b^{2} l^{-2} .
\end{aligned}
$$

The metric satisfies $R_{\mu \nu}=-4 l^{-2} g_{\mu \nu}$.

The outer event horizon is located at the largest positive root $r=r_{+}$of $\Delta=0$. It has area $A$ given by

$$
A=\frac{2 \pi^{2}\left(r_{+}^{2}+a^{2}\right)\left(r_{+}^{2}+b^{2}\right)}{r_{+} \Xi_{a} \Xi_{b}} .
$$

The surface gravity $\kappa$ is given by

$$
\kappa=r_{+}\left(1+r_{+}^{2} l^{-2}\right)\left(\frac{1}{r_{+}^{2}+a^{2}}+\frac{1}{r_{+}^{2}+b^{2}}\right)-\frac{1}{r_{+}} .
$$

The Hawking temperature is $T=1 / \beta=\kappa /(2 \pi)$.

The angular velocities, relative to a non-rotating frame at infinity, are given by

$$
\Omega_{a}=\frac{a\left(1+r_{+}^{2} l^{-2}\right)}{r_{+}^{2}+a^{2}}, \quad \Omega_{b}=\frac{b\left(1+r_{+}^{2} l^{-2}\right)}{r_{+}^{2}+b^{2}}
$$


The action of the five-dimensional Kerr-AdS metric is given by [1]

$$
I_{5}=\frac{\pi \beta}{4 \Xi_{a} \Xi_{b}}\left[m-l^{-2}\left(r_{+}^{2}+a^{2}\right)\left(r_{+}^{2}+b^{2}\right)\right]
$$

This result has been obtained using the background subtraction method, in which one regularises the infra-red divergence associated with the infinite volume by subtracting the action of AdS, with a boundary at large distance $R$ matched to the boundary of Kerr$\mathrm{AdS}$, and then sends $R$ to infinity. In general this procedure differs from the Brown-York procedure, widely used in AdS/CFT studies, in which one calculates a regularised action using a particular conformal choice of metric representative on the conformal boundary. The answer one obtains using the Brown-York procedure depends non-trivially on the choice of conformal representative. (See Appendix A for a discussion of the derivation of the KerrAdS action using the background subtraction method.)

There is even less agreement in $D=5$ than there was in $D=4$ about the correct expressions for the mass or energy $E$, and the angular momenta. However, as in four dimensions the evaluation of Komar integrals for the angular momenta is unambiguous. Again, for the mass one encounters a divergence in the Komar integral, with all the associated possibilities for ambiguity in extracting a meaningful finite result by subtraction.

The formulae for mass and angular momentum that we shall adopt are as follows:

$$
E=\frac{\pi m\left(2 \Xi_{a}+2 \Xi_{b}-\Xi_{a} \Xi_{b}\right)}{4 \Xi_{a}^{2} \Xi_{b}^{2}}, \quad J_{a}=\frac{\pi m a}{2 \Xi_{a}^{2} \Xi_{b}}, \quad J_{b}=\frac{\pi m b}{2 \Xi_{b}^{2} \Xi_{a}}
$$

The angular momenta are those that result from Komar integrals. We have arrived at our formula for the mass by thermodynamic considerations, as we shall now describe.

The first law of thermodynamics should now take the form

$$
d E=T d S+\Omega_{a} d J_{a}+\Omega_{b} d J_{b}
$$

since there are now two independent angular momenta in orthogonal transverse 2-planes. It is straightforward to verify that with $J_{a}$ and $J_{b}$ given in (3.7), and $T, \Omega_{a}$ and $\Omega_{b}$ as given above, then the right-hand side of (3.8) is indeed an exact differential, if the entropy is taken to be

$$
S=\frac{1}{4} A
$$

It can thus be integrated to give an energy function and, with the constant of integration chosen so that $E=0$ for the case $m=0$ of pure $\mathrm{AdS}_{5}$, the energy is precisely the one given in $(3.7)$. 
As a consistency check on our proposal in (3.7) for the mass $E$, we can easily verify that it indeed satisfies the Quantum Statistical Relation, which reads

$$
E-T S-\Omega_{a} J_{a}-\Omega_{b} J_{b}=T I_{5} .
$$

Alternatively, we could have started with the action $I_{5}$ given in (3.6), expressed it as a function of $\left(\beta, \Omega_{a}, \Omega_{b}\right)$, and then taken the thermodynamic potential to be $\Phi=I_{5} / \beta$. From this we could calculate the entropy and angular momenta. Doing so, one recovers the results that $S=\frac{1}{4} A$ and that the angular momenta are given by (3.7).

\subsection{Comparison with other literature}

Hawking, Hunter and Taylor-Robinson [1] gave the following expressions for the mass and the angular momenta of the five-dimensional Kerr-AdS black holes:

$$
E^{\prime}=\frac{3 \pi m}{4 \Xi_{a} \Xi_{b}}, \quad J_{a}^{\prime}=\frac{\pi m a}{2 \Xi_{a}^{2}}, \quad J_{b}^{\prime}=\frac{\pi m b}{2 \Xi_{b}^{2}} .
$$

They associate their angular momenta with angular velocities $\Omega_{a}^{\prime}$ and $\Omega_{b}^{\prime}$ on the horizon, which are defined relative to a frame at infinity that is rotating:

$$
\Omega_{a}^{\prime}=\frac{a \Xi_{a}}{r_{+}^{2}+a^{2}}, \quad \Omega_{b}^{\prime}=\frac{b \Xi_{b}}{r_{+}^{2}+b^{2}} .
$$

It will be seen that $E^{\prime}, J_{a}^{\prime}$ and $J_{b}^{\prime}$ all differ from the expressions (3.7) that we have adopted. The quantities $\left(E^{\prime}, J_{a}^{\prime}, J_{b}^{\prime}\right)$ satisfy neither a Quantum Statistical Relation, nor the first law of thermodynamics:

$$
\begin{aligned}
& d E^{\prime} \neq T d S+\Omega_{a}^{\prime} d J_{a}^{\prime}+\Omega_{b}^{\prime} d J_{b}^{\prime}, \\
& E^{\prime}-T S-\Omega_{a}^{\prime} J_{a}^{\prime}-\Omega_{b}^{\prime} J_{b}^{\prime} \neq T I_{5} .
\end{aligned}
$$

It can also be verified that the right-hand side of (3.13) is not an exact differential, and thus it cannot be integrated to get any energy function. ${ }^{7}$

It is worth remarking that although there have been various different proposals for the mass, most of the literature on the five-dimensional Kerr-AdS solution agrees that the angular momenta should be given by $J_{a}$ and $J_{b}$ in (3.7), rather than $J_{a}^{\prime}$ and $J_{b}^{\prime}$ in (3.11). If one adopts $J_{a}$ and $J_{b}$ for the angular momenta, but still follows the four-dimensional philosophy of [1] by using $\Omega_{a}^{\prime}$ and $\Omega_{b}^{\prime}$ for the angular velocities, then the mass $E^{\prime}$ proposed

\footnotetext{
${ }^{7}$ These statements would also be true if one replaced $\Omega_{a}^{\prime}$ and $\Omega_{b}^{\prime}$ by $\Omega_{a}$ and $\Omega_{b}$ in (3.13) and (3.14).
} 
by Hawking, Hunter and Taylor-Robinson does satisfy a Quantum Statistical Relation, namely

$$
E^{\prime}-T S-\Omega_{a}^{\prime} J_{a}-\Omega_{b}^{\prime} J_{b}=T I_{5}
$$

However, one again finds that the first law fails,

$$
d E^{\prime} \neq T d S+\Omega_{a}^{\prime} d J_{a}+\Omega_{b}^{\prime} d J_{b}
$$

and again, the right-hand side is not even an exact differential.

Berman and Parikh [19] discuss the thermodynamics of five-dimensional Kerr-AdS black holes with a single rotation parameter, giving expressions that coincide with those of Hawking, Hunter and Taylor-Robinson in this special case. In fact the angular momentum agrees with our expression in (3.7) in this special case (because, for example, if $b=0$ then $\Xi_{b}=1$ ). However, the mass does not agree with our expression in (3.7) even in this special case. Their discussion of the quantum statistical relation is similar to that of Hawking, Hunter and Taylor-Robinson, and works out for the same reason. However, just as in the case of Hawking, Hunter and Taylor-Robinson, they give no discussion of the first law, and in fact one does not obtain an exact differential on the right-hand side of (3.16).

The moral to be extracted from the above seems to be that one should always use the angular velocities measured relative to a non-rotating frame at infinity when discussing the thermodynamics of rotating black holes.

In a subsequent paper, Hawking and Reall [20] give expressions for the mass and angular momenta, for which they refer to [1]. However, although the mass coincides with the expression appearing in [1], and reproduced as $E^{\prime}$ in (2.16), the angular momenta differ from those appearing in [1] by a denominator factor of $\left(1+r_{+}^{2} l^{-2}\right)$.

Another proposal for the mass of the five-dimensional Kerr-AdS black hole is given by Awad and Johnson [21]. Based on the application of the Brown-York procedure, they obtain a mass

$$
E^{\prime \prime}=\frac{\pi l^{2}}{96 \Xi_{a} \Xi_{b}}\left(7 \Xi_{a} \Xi_{b}+\Xi_{a}^{2}+\Xi_{b}^{2}+72 m l^{-2}\right)
$$

which does not vanish in $\mathrm{AdS}_{5}$ spacetime $(m=0)$. This reduces in the non-rotating case to the expression obtained by Balasubramanian and Kraus [22]

$$
\frac{3 \pi l^{2}}{32}+\frac{3 \pi m}{4}
$$

In the AdS/CFT correspondence, the first term is attributed to the Casimir energy of the conformal field theory on the boundary. 
Awad and Johnson also present an expression for the action of the five-dimensional Kerr-AdS black hole,

$$
\begin{aligned}
I_{5}^{\prime \prime}=-\frac{\pi \beta l^{2}}{96 \Xi_{a} \Xi_{b}} & {\left[12 r_{+}^{2} l^{-2}\left(1-\Xi_{a}-\Xi_{b}\right)+\Xi_{a}^{2}+\Xi_{b}^{2}+\Xi_{a} \Xi_{b}+12 r_{+}^{4} l^{-4}\right.} \\
& \left.-2\left(a^{4}+b^{4}\right) l^{-4}-4 a^{2} b^{2} l^{-4}\left(3 r_{+}^{-2} l^{2}-1\right)-12\right] .
\end{aligned}
$$

This is obtained by a boundary counterterm subtraction procedure. Not surprisingly, this expression differs from the canonical expression (3.6) for the action.

One can easily verify that indeed, as stated in [21], the mass $E^{\prime \prime}$ given in (3.17) rather non-trivially satisfies a Quantum Statistical Relation, namely

$$
E^{\prime \prime}-T S-\Omega^{\prime} J_{a}-\Omega_{b}^{\prime} J_{b}=T I_{5}^{\prime \prime}
$$

It should be noted that the generally-accepted angular momenta $J_{a}$ and $J_{b}$ defined in (3.7) are being employed here, but the angular velocities are the ones given in (3.12), which are defined with respect to a frame rotating at infinity. The first law of thermodynamics is not investigated in [21], but it is easy to see that it is not satisfied;

$$
d E^{\prime \prime} \neq T d S-\Omega_{a}^{\prime} d J_{a}-\Omega_{b}^{\prime} d J_{b}
$$

Indeed, as we already observed, the right-hand side of this expression is not an exact differential, and so one could not achieve an equality in (3.21) for any choice of mass $E^{\prime \prime}$.

There is a further puzzling feature of Awad and Johnson's action $I=I_{5}^{\prime \prime}$. If one considers it as a function of the inverse temperature $\beta$, and angular velocities $\left(\Omega_{a}, \Omega_{b}\right)$ (or $\left(\Omega_{a}^{\prime}, \Omega_{b}^{\prime}\right)$ ), and calculates the energy $E$, entropy $S$ and angular momenta $\left(J_{a}, J_{b}\right)$ assuming that the quantum statistical relation holds,

$$
\begin{aligned}
E & =\frac{\partial I}{\partial \beta}-\frac{\Omega_{a}}{\beta} \frac{\partial I}{\partial \Omega_{a}}-\frac{\Omega_{b}}{\beta} \frac{\partial I}{\partial \Omega_{b}} \\
S & =\beta \frac{\partial I}{\partial \beta}-I, \quad J_{a}=-\frac{1}{\beta} \frac{\partial I}{\partial \Omega_{a}}, \quad J_{b}=-\frac{1}{\beta} \frac{\partial I}{\partial \Omega_{b}}
\end{aligned}
$$

one does not obtain their expression (3.17) for the energy, their (correct) expression $S=\frac{1}{4} A$ for the entropy, or their (correct) expression for the angular momenta $\left(J_{a}, J_{b}\right)$. Thus it seems that not only does the thermodynamics of the boundary system that they consider differ from the thermodynamics of the black holes in the bulk, but integrating the boundary stress tensor à la Brown-York, as they do, does not give the same expressions for the energy and angular momenta as one would obtain directly by interpreting the action as a thermodynamic potential. In any event, it is clear that unlike the expression $I_{5}$ for 
the action, given in (3.6), their action $I_{5}^{\prime \prime}$, given in (3.19), cannot be used to obtain the thermodynamic potential of the bulk black hole. We shall comment on this further in the next subsection.

It is perhaps worth emphasising that the differences one encounters between the various results in the literature typically involve terms depending upon the dimensionless ratios of the rotation parameters divided by the AdS radius $l$ (for example, via the quantities $\Xi_{a}$ and $\Xi_{b}$ ), which become irrelevant in the limit where the cosmological constant vanishes. Likewise, the differences between the angular velocities (3.5) and (3.12) disappear in the zero cosmological constant limit.

\subsection{Conformal infinity in the Hawking-Hunter-Taylor-Robinson metrics}

In this subsection, we discuss conformal infinity in the Hawking-Hunter-Taylor-Robinson metrics, emphasising in particular that different choices of conformal factor give rise to different metrics on the conformal boundary.

We begin by applying the transformation given in [1] that converts to asymptotically "canonical" AdS coordinates. In our notation, the new coordinates will be called $(y, \hat{\theta}, \hat{\phi}, \hat{\psi})$, related to the original coordinates $(r, \theta, \phi, \psi)$ of $(3.1)$ by

$$
\begin{aligned}
\Sigma_{a} y^{2} \sin ^{2} \hat{\theta} & =\left(r^{2}+a^{2}\right) \sin ^{2} \theta, \\
\Sigma_{b} y^{2} \cos ^{2} \hat{\theta} & =\left(r^{2}+b^{2}\right) \cos ^{2} \theta \\
\hat{\phi} & =\phi+a l^{-2} t, \\
\hat{\psi} & =\psi+b l^{-2} t .
\end{aligned}
$$

We find that the metric has the following asymptotic form, in terms of the new coordinates:

$$
\begin{aligned}
d s^{2}= & -\left(1+y^{2} l^{-2}\right) d t^{2}+\frac{d y^{2}}{1+y^{2} l^{-2}-\frac{2 m}{\Delta_{\hat{\theta}}^{2} y^{2}}}+y^{2} d \hat{\Omega}_{3}^{2} \\
& +\frac{2 m}{\Delta_{\hat{\theta}}^{3} y^{2}}\left(d t-a \sin ^{2} \hat{\theta} d \hat{\phi}-b \cos ^{2} \hat{\theta} d \hat{\psi}\right)^{2}+\cdots,
\end{aligned}
$$

where

$$
\begin{aligned}
\Delta_{\hat{\theta}} & \equiv 1-a^{2} l^{-2} \sin ^{2} \hat{\theta}-b^{2} l^{-2} \cos ^{2} \hat{\theta} \\
d \hat{\Omega}_{3}^{2} & \equiv d \hat{\theta}^{2}+\sin ^{2} \hat{\theta} d \hat{\phi}^{2}+\cos ^{2} \hat{\theta} d \hat{\psi}^{2}
\end{aligned}
$$

We recall that a bulk spacetime $\{X, g\}$ with conformal boundary $\{\partial X, \bar{h}\}$ admits a conformal compactification $\{\bar{X}, \bar{g}\}$ if $\bar{X}=X \sqcup \partial X$ is the closure of $X$ and the metric $\bar{g}$ 
extends smoothly to $\bar{X}$ with $\bar{g}=\Omega^{2} g$ for some function $\Omega$ with $\Omega>0$ in $X$ and $\Omega=0$ on $\partial X$, with $d \Omega \neq 0$ on $\partial X$. Since $\Omega$ is determined only up to a factor, $\Omega \rightarrow f \Omega$, where the function $f$ is non-zero on $\partial X$, the metric $\bar{g}$ on $\bar{X}$ and its restriction $\bar{h}=\left.\bar{g}\right|_{\partial X}$ are defined only up to a conformal factor. The conformal equivalence class $\{\partial \bar{X}, \bar{h}\}$ is called the conformal boundary of $X$.

In our case, we may take

$$
\Omega=\frac{l}{y},
$$

so that $\partial X$ is given by $y=\infty$ and a short calculation shows that $\bar{h}$ for this choice of conformal factor is given by

$$
d \bar{s}^{2}=-d t^{2}+l^{2} d \Omega_{3}^{2}
$$

This is the standard metric on the Einstein static universe, or, equivalently, on the conformal compactification of four-dimensional Minkowski spacetime.

Working in the $(y, \hat{\theta}, \hat{\phi}, \hat{\psi})$ coordinates is rather clumsy, and we may instead use the Boyer-Lindquist coordinates $(r, \theta, \phi, \psi)$ of equation (3.1). In this case it is natural to choose

$$
\Omega=\frac{l}{r},
$$

for which one finds that the metric on the conformal boundary is given by

$$
d \bar{s}^{\prime 2}=-d t^{2}+\frac{2 a \sin ^{2} \theta}{\Xi_{a}} d t d \phi+\frac{2 b \cos ^{2} \theta}{\Xi_{b}} d t d \psi+\frac{l^{2} d \theta^{2}}{\Delta_{\theta}}+\frac{l^{2} \sin ^{2} \theta}{\Xi_{a}} d \phi^{2}+\frac{l^{2} \cos ^{2} \theta}{\Xi_{b}} d \psi^{2} .
$$

Using the coordinate transformations

$$
\tan ^{2} \theta=\frac{\Xi_{a}}{\Xi_{b}} \tan ^{2} \hat{\theta}, \quad \phi=\Phi-a l^{-2} t, \quad \psi=\Psi-b l^{-2} t,
$$

we find that the two boundary metrics (3.31) and (3.33) are indeed conformally related, with

$$
d{\overline{s^{\prime}}}^{2}=\frac{1}{\Delta_{\hat{\theta}}} d \bar{s}^{2} .
$$

In fact, this conformal factor relating the two boundary metrics is essentially given by $\Delta_{\hat{\theta}}^{-1}=y^{2} / r^{2}$.

An important point is that the static boundary metric $d{\overline{s^{\prime}}}^{2}$ has $g_{t t}=-1 / \Delta_{\hat{\theta}}$. This means that in thermal equilibrium the temperature will be $\hat{\theta}$ dependent, because of Tolman's wellknown red-shifting law, $T \sqrt{-g_{t t}}=$ constant [23]. In the present case, this tells us that the local temperature $T=T(\hat{\theta})$ is given by

$$
T(\hat{\theta})=T_{0} \sqrt{\Delta_{\hat{\theta}}}
$$


where $T_{0}$ is a constant. This spatial dependence of the equilibrium temperature may be responsible for the discrepancies found in section 3.2 when we compared the energy and angular momenta given by Awad and Johnson with the expressions that follow from differentiating their action, as in (3.22).

It is also important to bear in mind, as emphasised by Skenderis [24], that if one uses the boundary stress tensor method to obtain actions, energies and angular momenta, these will in general depend upon the choice of conformal representative for the boundary metric, if the spacetime dimension is odd. This is because in odd spacetime dimensions, the (evendimensional) boundary is subject to conformal anomalies. In particular, calculations carried out using the conformal factor $\Omega=l / r$, which may seem natural and convenient in the Boyer-Lindquist coordinates $(r, \theta, \phi, \psi)$, will give different answers from those carried out using the conformal factor $\Omega=l / y$ that is natural in the $(y, \hat{\theta}, \hat{\phi}, \hat{\psi})$ coordinates. It may be that this accounts for some of the differences between the formulae of Awad and Johnson [21] (who use $\Omega=l / r$ ) and those of other authors. As Ashtekar and Das have emphasised [25], there is no way of reconciling the $S O(4,2)$ anti-de Sitter symmetry with an energy, such as that of $[21,22]$, that is non-vanishing in pure AdS spacetime. We ourselves have not used the boundary stress tensor procedure, and have endeavoured to maintain bulk diffeomorphism invariance throughout.

\section{Kerr-AdS Black Holes in $D \geq 6$ Dimensions}

\subsection{Thermodynamics in $D \geq 6$ Kerr-AdS}

The general Kerr-de Sitter metrics in arbitrary dimension $D$ were obtained in [2]. They have $N \equiv[(D-1) / 2]$ independent rotation parameters $a_{i}$ in $N$ orthogonal 2-planes. We have $D=2 N+1$ when $D$ is odd, and $D=2 N+2$ when $D$ is even. Defining $\epsilon \equiv(D-1)$ $\bmod 2$, so that $D=2 N+1+\epsilon$, the metrics can be described by introducing $N$ azimuthal angles $\phi_{i}$, and $(N+\epsilon)$ "direction cosines" $\mu_{i}$ obeying the constraint

$$
\sum_{i=1}^{N+\epsilon} \mu_{i}^{2}=1 .
$$

In Boyer-Linquist coordinates, the metrics are given by [2]

$$
\begin{aligned}
d s^{2}= & -W\left(1+r^{2} l^{-2}\right) d \tau^{2}+\frac{2 m}{U}\left(W d \tau-\sum_{i=1}^{N} \frac{a_{i} \mu_{i}^{2} d \varphi_{i}}{\Xi_{i}}\right)^{2}+\sum_{i=1}^{N} \frac{r^{2}+a_{i}^{2}}{\Xi_{i}} \mu_{i}^{2} d \varphi_{i}^{2} \\
& +\frac{U d r^{2}}{V-2 m}+\sum_{i=1}^{N+\epsilon} \frac{r^{2}+a_{i}^{2}}{\Xi_{i}} d \mu_{i}^{2}-\frac{l^{-2}}{W\left(1+r^{2} l^{-2}\right)}\left(\sum_{i=1}^{N+\epsilon} \frac{r^{2}+a_{i}^{2}}{\Xi_{i}} \mu_{i} d \mu_{i}\right)^{2}
\end{aligned}
$$


where

$$
\begin{aligned}
W & \equiv \sum_{i=1}^{N+\epsilon} \frac{\mu_{i}^{2}}{\Xi_{i}}, \quad U \equiv r^{\epsilon} \sum_{i=1}^{N+\epsilon} \frac{\mu_{i}^{2}}{r^{2}+a_{i}^{2}} \prod_{j=1}^{N}\left(r^{2}+a_{j}^{2}\right), \\
V & \equiv r^{\epsilon-2}\left(1+r^{2} l^{-2}\right) \prod_{i=1}^{N}\left(r^{2}+a_{i}^{2}\right), \quad \Xi_{i} \equiv 1-a_{i}^{2} l^{-2} .
\end{aligned}
$$

They satisfy $R_{\mu \nu}=-(D-1) l^{-2} g_{\mu \nu}$.

The outer horizon is located at $r=r_{+}$, where $r_{+}$is the largest root of $V(r)-2 m=0$. The surface gravity $\kappa$ and the area $A$ of the event horizon are given by [2]

$$
\begin{aligned}
D=\text { odd }: \quad \kappa & =r_{+}\left(1+r_{+}^{2} l^{-2}\right) \sum_{i} \frac{1}{r_{+}^{2}+a_{i}^{2}}-\frac{1}{r_{+}}, \\
A & =\frac{\mathcal{A}_{D-2}}{r_{+}} \prod_{i} \frac{r_{+}^{2}+a_{i}^{2}}{\Xi_{i}} \\
D=\text { even }: \quad \kappa & =r_{+}\left(1+r_{+}^{2} l^{-2}\right) \sum_{i} \frac{1}{r_{+}^{2}+a_{i}^{2}}-\frac{1-r_{+}^{2} l^{-2}}{2 r_{+}}, \\
A & =\mathcal{A}_{D-2} \prod_{i} \frac{r_{+}^{2}+a_{i}^{2}}{\Xi_{i}}
\end{aligned}
$$

where $\mathcal{A}_{D-2}$ is the volume of the unit $(D-2)$-sphere:

$$
\mathcal{A}_{D-2}=\frac{2 \pi^{(D-1) / 2}}{\Gamma[(D-1) / 2]} .
$$

The Hawking temperature is then given by $T=\kappa /(2 \pi)$. The angular velocities, measured relative to a frame that is non-rotating at infinity, are given by

$$
\Omega_{i}=\frac{\left(1+r_{+}^{2} l^{-2}\right) a_{i}}{r_{+}^{2}+a_{i}^{2}} .
$$

By evaluating Komar integrals,

$$
J_{i} \sim \int_{S^{D-2}} * d K_{i}
$$

where $K_{i}=\frac{\partial}{\partial \phi^{i}}$ are the angular Killing vectors, one one can see that the angular momenta should be given in terms of the rotation parameters by

$$
J_{i}=\frac{m a_{i} \mathcal{A}_{D-2}}{4 \pi \Xi_{i}\left(\prod_{j} \Xi_{j}\right)}
$$

These expressions agree with those in (2.12) and (3.7) in four and five dimensions. We have explicitly evaluated the Komar integrals in dimensions $D \leq 7$. 
We now consider the first law of thermodynamics, which will read

$$
d E=T d S+\sum_{i} \Omega_{i} d J_{i}
$$

It can be verified that the right-hand side is indeed an exact differential, if the expressions (4.12) are used for the angular momenta, and if the entropy is taken to be

$$
S=\frac{1}{4} A
$$

This provides a non-trivial check of the correctness of (4.12). We can then integrate (4.13), thereby allowing us to learn the mass $E$ of the black hole. We find

$$
\begin{array}{ll}
D=\text { odd }: & E=\frac{m \mathcal{A}_{D-2}}{4 \pi\left(\prod_{j} \Xi_{j}\right)}\left(\sum_{i=1}^{N} \frac{1}{\Xi_{i}}-\frac{1}{2}\right) \\
D=\text { even }: & E=\frac{m \mathcal{A}_{D-2}}{4 \pi\left(\prod_{j} \Xi_{j}\right)} \sum_{i=1}^{N} \frac{1}{\Xi_{i}}
\end{array}
$$

It is easily seen that in the cases $D=4$ and $D=5$, these expressions reduce to the ones given in (2.12) and (3.7) respectively. In section 5, we shall calculate the masses of the general rotating AdS black holes using the Ashtekar-Magnon-Das conformal mass definition, and show that they are in agreement with our expressions (4.15) and (4.16).

By applying the standard background-subtraction procedure for calculating the Euclidean action $I_{D}$ of the $D$-dimensional Kerr-AdS metric, described in detail in Appendix A, we find that it is given by

$$
\begin{array}{ll}
D=\text { odd }: & I_{D}=\frac{\beta \mathcal{A}_{D-2}}{8 \pi\left(\prod_{j} \Xi_{j}\right)}\left(m-l^{-2} \prod_{i=1}^{N}\left(r_{+}^{2}+a_{i}^{2}\right)\right) \\
D=\text { even }: & I_{D}=\frac{\beta \mathcal{A}_{D-2}}{8 \pi\left(\prod_{j} \Xi_{j}\right)}\left(m-r_{+} l^{-2} \prod_{j=i}^{N}\left(r_{+}^{2}+a_{i}^{2}\right)\right)
\end{array}
$$

It is straightforward to verify that with our results (4.12), (4.15) and (4.16) for the angular momenta and masses of the Kerr-AdS black holes, the Quantum Statistical Relation

$$
E-T S-\sum_{i} \Omega_{i} J_{i}=T I_{D}
$$

holds. 


\subsection{Comparison with other literature}

No very complete comparisons with previous results can be made in dimensions $D \geq 6$, since the general rotating Kerr-AdS solutions were not available until recently. The metrics were obtained in [1] for the special case where all except one rotation parameter vanishes, and so some comparisons in these restricted cases are possible.

Awad and Johnson [21] give expressions in $D=6$ for the mass, angular momentum and action when (to take a concrete choice for the specialisation) $a_{1} \neq 0, a_{2}=0$. They give

$$
E^{\prime}=\frac{4 \pi m}{3 \Xi_{1}}, \quad J_{1}=\frac{2 \pi m a_{1}}{3 \Xi_{1}^{2}}
$$

and an expression for the action $I_{6}$ that is easily verified to be the same as the specialisation

of (4.18) to $D=6, a_{2}=0$ (after inserting a factor of $\frac{1}{3}$ in their expression whose absence is presumably due to a typographical error.). Their expression for $J_{1}$ also agrees with our general result (4.12), under the specialisation $a_{2}=0$. Their expression $E^{\prime}$ for the mass, however, disagrees with the specialisation of (4.16) to $D=6, a_{2}=0$, which would give

$$
E=\frac{2 \pi m}{3 \Xi_{1}}\left(1+\frac{1}{\Xi_{1}}\right)
$$

As observed in [21], their expression for $E^{\prime}$ satisfies the Quantum Statistical Relation

$$
E^{\prime}-T S-\Omega_{1}^{\prime} J_{1}=T I_{6},
$$

where the angular velocity $\Omega_{1}^{\prime}=a_{1} \Xi_{1} /\left(r_{+}^{2}+a_{1}^{2}\right)$ is defined with respect to a frame that is rotating at infinity. However, as usual when such an angular velocity is employed, we find that the first law is not satisfied,

$$
d E^{\prime} \neq T d S+\Omega_{1}^{\prime} d J_{1}
$$

and furthermore the right-hand side is not an exact differential.

Awad and Johnson have also studied Kerr-AdS black holes in $D=7$, in the special case where two of the three rotation parameters vanish, say $a_{2}=a_{3}=0$. They give the mass and angular momentum as

$$
E^{\prime}=-\frac{\pi^{2} l^{4}\left(a_{1}^{6} l^{-6}+5 a_{1}^{4} l^{-4}+50 \Xi_{1}-800 m l^{-4}\right)}{1280 \Xi_{1}}, \quad J_{1}=\frac{\pi^{2} m a_{1}}{4 \Xi_{1}^{2}}
$$

and an expression for the action $I_{7}^{\prime}$ where, as in $D=5$, they employ a counterterm subtraction scheme. Their expression for the angular momentum agrees with our general result (4.12), specialised to $D=7$ and $a_{2}=a_{3}=0$. However, their expressions for the mass and 
the action disagree with ours. They again find that their expressions satisfy a Quantum Statistical Relation,

$$
E^{\prime}-T S-\Omega_{1}^{\prime} J_{1}=T I_{7}^{\prime}
$$

Although they do not address the issue, it is easy to see that

$$
d E^{\prime} \neq T d S+\Omega_{1}^{\prime} d J_{1}
$$

and so the first law is not satisfied, and furthermore the right-hand side of (4.26) is not an exact differential.

\section{Thermodynamic Energy and the Conformal Mass}

Astekar and Magnon [13] and Ashtekar and Das [25] have given a conformal definition of a conserved quantity $Q[K]$ associated to an asymptotic Killing field $K$ in an aymptotically anti-de Sitter $D$-dimensional spacetime. The expression for $Q[K]$ is linear in $K$ and involves an integral of certain components of the Weyl tensor over a codimension-2 sphere lying on the conformal boundary. Specifically, if $\bar{C}^{\mu}{ }_{\nu \rho \sigma}$ is the Weyl tensor of the conformally rescaled metric $\bar{g}_{\mu \nu}=\Omega^{2} g_{\mu \nu}, \bar{n}_{\mu}=\partial_{\mu} \Omega$ and

$$
\overline{\mathcal{E}}^{\mu}{ }_{\nu}=l^{2} \Omega^{D-3} \bar{n}^{\rho} \bar{n}^{\sigma} \bar{C}_{\rho \nu \sigma}^{\mu}
$$

is the electric part of the Weyl tensor on the conformal boundary, then $Q[K]$ is given by ${ }^{8}$

$$
Q[K]=\frac{l}{8 \pi(D-3)} \oint_{\Sigma} \overline{\mathcal{E}}^{\mu}{ }_{\nu} K^{\nu} d \bar{\Sigma}_{\mu},
$$

where $d \bar{\Sigma}_{\mu}$ is the area element of the $(D-2)$-sphere section of the conformal boundary.

If $K_{1}$ and $K_{2}$ are two asymptotic Killing vector fields, then $a K_{1}+b K_{2}$ is also an asymptotic Killikng vector field, and clearly

$$
Q\left[c_{1} K_{1}+c_{2} K_{2}\right]=c_{1} Q\left[K_{1}\right]+c_{2} Q\left[K_{2}\right]
$$

where $c_{1}$ and $c_{2}$ are constants. Theses charges were evaluated by Das and Mann [27] for the angular momentum and energy associated to the Killing fields $\partial / \partial \phi$ and $\partial / \partial t$ in Boyer-Lindquist coordinates, in the case of the Kerr-AdS solutions with only one non-zero rotation parameter, in $4 \leq D \leq 9$ spacetime dimensions. They compared their results with those obtained using the boundary stress-tensor (counterterm) on the spheroidal surfaces $r=$ constant. The angular momenta agree with those coming from the boundary stress

\footnotetext{
${ }^{8}$ Our sign for $Q[K]$ differs from that in [25]; we use orientation conventions such that $d \bar{\Sigma}_{t}$ is positive.
} 
tensor in all dimensions, and they agree with those given in this paper (after specialising our results to a single non-vanishing rotation parameter). They obtained agreement for the energy in even spacetime dimensions, but their results disagreed in odd spacetime dimensions. The conformal energies given in [27] also differ from our expressions (after specialising our results to a single non-vanishing rotation parameter).

Das and Man attributed the discrepancy between their conformal energy and the counterterm expression to a Casimir energy. However, they calculated the energy using the Killing field $K \equiv \partial / \partial t$, which is rotating even at infinity. The non-rotating timelike Killing field is

$$
\frac{\partial}{\partial t}+\frac{a}{l^{2}} \frac{\partial}{\partial \phi}
$$

Thus the conformal energy with respect to it is

$$
Q\left[\partial_{t}+a l^{-2} \partial_{\phi}\right]=Q\left[\partial_{t}\right]+\frac{a}{l^{2}} Q\left[\partial_{\phi}\right]
$$

where $\partial_{t} \equiv \partial / \partial t$ and $\partial_{\phi} \equiv \partial / \partial \phi$. In all cases $Q\left[\partial_{\phi}\right]=J$, and so

$$
Q\left[\partial_{t}+a l^{-2} \partial_{\phi}\right]=Q\left[\partial_{t}\right]+\frac{a}{l^{2}} J
$$

If one uses the values for $Q\left[\partial_{t}\right]$ provided in [27], one in fact finds that

$$
Q\left[\partial_{t}+a l^{-2} \partial_{\phi}\right]=E,
$$

where $E$ is our expression for the thermodynamic mass, specialised to the case of a single non-zero rotation parameter.

We shall now generalise this result to the case with arbitrary non-zero rotation parameters. The conformal mass, which should be calculated using the timelike Killing vector that is non-rotating at infinity, is given by

$$
M_{\mathrm{conf}} \equiv Q\left[\partial_{t}+\sum_{i} a_{i} l^{-2} \partial_{\phi_{i}}\right]=Q\left[\partial_{t}\right]+\sum_{i=1}^{N} \frac{a_{i}}{l^{2}} J_{i}
$$

To evaluate $Q\left[\partial_{t}\right]$, we note that the leading-order term in the expression for the coordinate component $C^{t}{ }_{r t r}$ of the Weyl tensor of the physical Kerr-AdS metric $g_{\mu \nu}$ given in (4.2) is

$$
C_{r t r}^{t}=\frac{m(D-2)(D-3) l^{2}}{r^{D+1}}+\cdots
$$

at large distance. The electric component $\overline{\mathcal{E}}^{t}{ }_{t}$, which is defined on the conformal boundary, is therefore given by

$$
\overline{\mathcal{E}}^{t}{ }_{t}=m l^{1-D}(D-2)(D-3) .
$$


From the expression (A.9) for the determinant of $g_{\mu \nu}$, we see that the volume element $d \bar{\Sigma}_{t}$ of the spacelike hypersurface $t=$ constant lying in the boundary is given by

$$
d \bar{\Sigma}_{t}=\frac{l^{D-2}}{\prod_{j} \Xi_{j}} d \Omega_{D-2},
$$

where $d \Omega_{D-2}$ is the volume element of the unit $(D-2)$-sphere. Substituting into (5.2) with $K=\partial / \partial t$, we therefore find that $Q\left[\partial_{t}\right]$ for the general Kerr-AdS metric in $D$ dimensions is given by

$$
Q\left[\partial_{t}\right]=\frac{m(D-2) \mathcal{A}_{D-2}}{8 \pi\left(\prod_{j} \Xi_{j}\right)} .
$$

Thus from (5.8) the conformal mass is given by

$$
M_{\mathrm{conf}}=\frac{m(D-2) \mathcal{A}_{D-2}}{8 \pi\left(\prod_{j} \Xi_{j}\right)}+\sum_{i=1}^{N} \frac{a_{i}}{l^{2}} J_{i} .
$$

Using (4.12), we therefore find that

$$
M_{\mathrm{conf}}=E
$$

where $E$ is given in (4.15) and (4.16). In other words, we have shown that our expression for the thermodynamic mass $E$ is indeed equal to the Ashtekar-Magnon-Das conformal mass $M_{\text {conf }}$ evaluated using the non-rotating asymptotic timelike Killing field

$$
\frac{\partial}{\partial t}+\sum_{i=1}^{N} \frac{a_{i}}{l^{2}} \frac{\partial}{\partial \phi_{i}}
$$

It is worth remarking that a great advantage of the Ashtekar-Magnon-Das definition of mass in an asymptotically AdS background is that the integral (5.2) directly gives a finite result, unlike the divergent expression one obtains using the Komar mass formula. Thus all the amiguities that plague the Komar prescription are avoided in the Ashtekar-Magnon-Das approach.

\section{Conclusions}

In this paper, we have discussed the thermodynamics of a rotating black hole in a background anti-de Sitter spacetime. ${ }^{9}$ Our work has been concerned entirely with the bulk theory, and we have maintained bulk diffeomorphism invariance throughout. In particular, we have maintained the full $S O(D-1,2)$ invariance. We have given expressions for the

\footnotetext{
${ }^{9}$ See [26] for a recent discussion of the thermodynamics of non-rotating charged AdS black holes.
} 
masses, angular momenta, and actions of the Kerr-AdS black hole solutions in all dimensions $D$. The actions were calculated using the background subtraction method. We find, in contrast to some earlier work, a complete consistency between our answers and the first-law of thermodynamics. In particular, as a result we confirm that the entropy of the black hole is given by $\frac{1}{4}$ of the area, a result that is frequently assumed, but seldom established. We also confirm that the bulk action $I$ and the the thermodynamic potential $\Phi$ are related at temperature $T$ by the Quantum Statistical Relation

$$
Z=e^{-\Phi / T}=e^{-I}
$$

We have also shown that our expression for the mass of a general Kerr-AdS black hole in $D$ dimensions coincides with the coincides with that given by the conformal conserved charge introduced by Ashtekar, Magnon and Das, provided one uses the timelike Killing vector field that is non-rotating at infinity.

Some of our results differ from those found in the literature, and we have tried to explain why this is the case. Much of the recent work on Kerr-AdS black holes was motivated by the conjectured AdS/CFT correspondence. In this context, in odd bulk spacetime dimensions there are conformal anomalies in the boundary theory, which break the bulk $S O(D-1,2)$ invariance, and moreover are dependent upon the choice of representative of the conformal structure on the conformal boundary.

Hawking, Hunter and Taylor-Robinson [1] suggested that the CFT dual of a KerrAdS black hole is a conformal field theory that is rotating in a background Einstein static universe. A comparison of their partition function with that of such a rotating gas, in the case of no interactions, revealed the same type of singularities as the rotation angular velocities tended to their maximum values, i.e. as $\Xi_{a}$ or $\Xi_{b}$ tended to zero. Follow-up studies by Hawking and Reall [20], Berman and Parikh [19], and Landsteiner and Lopez [28], showed that for fixed angular velocities but high temperatures, the thermodynamic potentials agreed at leading order (modulo the standard factor of $\frac{3}{4}$ ), but they differed at sub-leading orders. Thus there appears to be a satisfactory agreement between the bulk and boundary theories, in the case that the metric on the boundary is the standard metric on the Einstein static universe. In the work of Awad and Johnson, the boundary metric was taken to be conformal to the standard Einstein static universe, and hence their results differ. It would be an interesting project to investigate in more detail the relationship between the bulk theory and these two boundary theories. 


\section{Acknowledgments}

We are grateful to Abhay Ashtekar for drawing our attention to reference [27], and for helpful discussions. G.W.G. and C.N.P. thank Boğaziçi University, Istanbul, for hospitality during the course of this work. C.N.P. is also grateful to the Relativity Group in DAMTP, Cambridge and the CERN Theory Division for hospitality. Many of the calculations in this paper have been performed with the aid of Mathematica.

\section{Note Added}

It has recently been shown in [29] that our expressions for the masses and angular momenta of the rotating AdS black holes can also be derived from the superpotential of Katz, Bičák and Lynden-Bell [30]. 


\section{Appendix}

\section{A Euclidean Action of the Kerr-AdS Metrics}

The Euclidean action $I_{D}$ of the $D$-dimensional Kerr-AdS metric can be calculated as follows. The metric satisfies the Einstein equation $R_{\mu \nu}=-(D-1) l^{-2} g_{\mu \nu}$, which can be derived from the action

$$
I_{D}^{\prime}=-\frac{1}{16 \pi} \int \sqrt{-g}\left[R+(D-1)(D-2) l^{-2}\right] d^{D} x .
$$

After Euclideanisation, achieved by sending $t \longrightarrow-\mathrm{i} \tau$ and $a_{j} \longrightarrow \mathrm{i} \alpha_{j}$, the metric extends smoothly onto the horizon at $r=r_{+}$(the largest positive root of $V(r)-2 M=0$ ) if $\tau$ is assigned the period $\beta=2 \pi / \kappa$, where $\kappa$ is the surface gravity. Thus the Euclidean action will be given by

$$
\begin{gathered}
I_{D}=-\frac{1}{16 \pi} \int_{\mathcal{M}} \sqrt{g}\left[R+(D-1)(D-2) l^{-2}\right] d^{D} x-\frac{1}{8 \pi} \int_{\partial \mathcal{M}} \sqrt{h} K d^{D-1} x, \\
=\frac{(D-1)}{8 \pi l^{2}} \int_{\mathcal{M}} \sqrt{g} d^{D} x-\frac{1}{8 \pi} \int_{\partial \mathcal{M}} \sqrt{h} K d^{D-1} x,
\end{gathered}
$$

where we have included the surface contribution, whose integrand $K$ is the trace of the second fundamental form of the boundary, and the Euclidean time coordinate $\tau$ is integrated over its period $\beta$.

As it stands, the expression (A.2) diverges because the volume of the Euclidean KerrAdS space is infinite. This divergence arises because $r$ is integrated from $r_{+}$to infinity. To regularise this expression, one terminates the $r$ integration at $r=R$, subtracts off the action for pure AdS adjusted so that its metric matches that of Kerr-AdS at $r=R$, and then sends $R$ to infinity. This regularisation prescription is a natural one since the Kerr-AdS metric with $m=0$ is nothing but AdS itself (expressed in non-standard "spheroidal" coordinates). Thus the regularised action of Kerr-AdS is its action measured relative to the action of the AdS "vacuum" that results from turning off the mass parameter $m$. It should be noted that the surface terms will cancel when the background subtraction is performed, and thus we need concentrate only on the bulk volume integrals.

The evaluation of the Euclidean action for the Kerr-AdS metric $d s^{2}$ itself, with the radial integration running from $r_{+} \leq r \leq R$ is straightforward. There are two subtleties that arise

in the subtraction of the action for the AdS metric $d \bar{s}^{2}$. Firstly, in order to match the two metrics on the surface $r=R$ at large $R$, one must rescale the Euclidean time coordinate $\tau$ 
appropriately. Thus the rescaled coordinate $\tau_{0}$ in the AdS metric is related to $\tau$ by

$$
(V(R)-2 m) \tau^{2}=V(R) \tau_{0}^{2}
$$

which, for large $R$, implies

$$
\tau_{0}=\left(1-\frac{m l^{2}}{R^{D-1}}\right) \tau
$$

and hence $\tau_{0}$ has period $\beta_{0}$ given by

$$
\beta_{0}=\left(1-\frac{m l^{2}}{R^{D-1}}\right) \beta
$$

The second subtlety concerns the integration over the volume of the Euclideanised AdS space. To understand this, we must look at the relation between the coordinate system used in writing the Kerr-AdS metric (4.2), and the coordinate system with respect to which the Kerr-AdS metric with $m=0$ (which is just AdS) becomes the AdS metric written in canonical coordinates. For general dimension $D$ this transformation is given in [2]: The "asymptotically-canonical" coordinates are obtained by replacing $\left(r, \mu_{i}\right)$ by $\left(y, \hat{\mu}_{i}\right)$, where $\sum_{i} \hat{\mu}_{i}^{2}=1$ and

$$
y^{2} \Xi_{i} \hat{\mu}_{i}^{2}=\left(r^{2}+a_{i}^{2}\right) \mu_{i}^{2}
$$

for each $1 \leq i \leq N+\epsilon$. It should be recalled that $N=[(D-1) / 2], \epsilon=(D-1) \bmod 2$, and so $D=2 N+\epsilon+1$. Recall also that when $D$ is even, there is no azimuthal angle $\phi_{N+1}$ associated with the direction cosine $\mu_{N+1}$, and correspondingly $a_{N+1} \equiv 0$. It is shown in [2] that under the transformation (A.6), the Kerr-AdS metric (4.2) with $m=0$ becomes

$$
d \bar{s}^{2}=-\left(1+y^{2} l^{-2}\right) d t^{2}+\frac{d y^{2}}{1+y^{2} l^{-2}}+y^{2} \sum_{k=1}^{N+\epsilon}\left(d \hat{\mu}_{k}^{2}+\hat{\mu}_{k}^{2} d \phi_{k}^{2}\right),
$$

which can be recognised as the standard metric on AdS.

Expressed in terms of $\left(y, \hat{\mu}_{i}\right)$, integration over the volume of AdS would involve integrating $y$ from 0 to some large value $y_{1}$. Care is needed at the lower end of the radial integration, $r=r_{0}$, where $y$ goes to zero. From (A.6), we see that if $D=2 N+2$, meaning that $\epsilon=1$ and $a_{N+1}=0$, then $y=0$ just corresponds to $r=r_{0}=0$. However, if $D=2 N+1$, and all $N$ of the rotation parameters $a_{i}$ are non-vanishing, we shall instead have that $y=0$ corresponds to $r=r_{0}$ with

$$
r_{0}^{2}=-\frac{\sum_{i} a_{i}^{2} \mu_{i}^{2} \Xi_{i}^{-1}}{\sum_{j} \mu_{j}^{2} \Xi_{j}^{-1}}
$$

and this determines the lower limit of the radial integration. (It will be imaginary, using the radial coordinate $r$.) If, however, the $a_{i}$ are not all non-vanishing, then $y=0$ will correspond instead to $r=0$. 
In order to perform the necessary integrations to calculate the action, it is helpful to record the expression for the volume element of the Kerr-AdS metrics (4.2). The $\mu_{i}$ coordinates are subject to the constraint (4.1), which can be used in order to solve for, say, $\mu_{N+\epsilon}$ in terms of the remaining $\mu_{\alpha}$, where $1 \leq \alpha \leq N+\epsilon-1$; i.e. $\mu_{N+\epsilon}=\sqrt{1-\sum_{\alpha} \mu_{\alpha}^{2}}$. (Note that in even dimensions, we choose to solve for the "extra" direction cosine $\mu_{N+1}$ that is not paired with an azimuthal angle.) Using these coordinates, we find

$$
\sqrt{-g}=\frac{r U \prod_{i=1}^{N} \mu_{i}}{\mu_{N+\epsilon} \prod_{j=1}^{N} \Xi_{j}} .
$$

Thus we have

$$
\begin{array}{ll}
D=2 N+1: & \sqrt{-g}=\frac{r U \prod_{i=1}^{N-1} \mu_{i}}{\prod_{j=1}^{N} \Xi_{j}} \\
D=2 N+2: & \sqrt{-g}=\frac{r U \prod_{i=1}^{N} \mu_{i}}{\mu_{N+1} \prod_{j=1}^{N} \Xi_{i}},
\end{array}
$$

where $U$ can be read off in each case from (4.3). It should also be noted that each direction cosine $\mu_{i}$ paired with an azimuthal coordinate $\phi_{i}$ ranges over $0 \leq \mu_{i} \leq 1$, but the extra unpaired direction cosine $\mu_{N+1}$ in the case that $D$ is even ranges over $-1 \leq \mu_{N+1} \leq 1$.

The regularised action $I_{D}$ is now evaluated by taking the limit as $R \rightarrow \infty$ of the difference $Y-Y_{0}$, where $Y$ is the action for the Kerr-AdS metric integrated to radius $R$,

$$
Y=\frac{(D-1) \beta}{8 \pi l^{2}} \int_{r_{+}}^{R} d r \int d^{D-2} x \sqrt{-g},
$$

and $Y_{0}$ is the action for the AdS metric integrated to radius $R$,

$$
Y_{0}=\frac{(D-1) \beta_{0}}{8 \pi l^{2}} \int_{r_{0}}^{R} d r \int d^{D-2} x \sqrt{-\bar{g}} .
$$

In these expressions, $d^{D-2} x$ represents the integration over the direction cosines $\mu_{\alpha}$ and the azimuthal angles $\phi_{i}$.

By considering specific cases, and evaluating

$$
I_{D}=\lim _{R \rightarrow \infty}\left(Y-Y_{0}\right)
$$

we find that the Euclidean action for the $D$-dimensional Kerr-AdS metric can be written as

$$
\begin{array}{ll}
D=\text { odd }: & I_{D}=\frac{\beta \mathcal{A}_{D-2}}{8 \pi\left(\prod_{j} \Xi_{j}\right)}\left(m-l^{-2} \prod_{i=1}^{N}\left(r_{+}^{2}+a_{i}^{2}\right)\right), \\
D=\text { even }: & I_{D}=\frac{\beta \mathcal{A}_{D-2}}{8 \pi\left(\prod_{j} \Xi_{j}\right)}\left(m-r_{+} l^{-2} \prod_{i=1}^{N}\left(r_{+}^{2}+a_{i}^{2}\right)\right),
\end{array}
$$


where $A_{D-2}$ is the volume of the unit $(D-2)$-sphere, given in (4.9). The integrations are relatively straightforward for the even-dimensional cases, for which the lower limit $r_{0}$ in the subtracted AdS action is $r_{0}=0$, but it is much more complicated in the odd-dimensional cases, due to the angle-dependent lower limit $r_{0}$ given by (A.8). We have explicitly evaluated the integrals leading to (A.15) for dimensions $D=5,7$ and 9; and the integrals leading to (A.16) for $D=4,6,8,10$ and 12 .

The explicit evaluation of the integrals becomes much easier in certain special cases. An especially simple case is when all the rotation parameters $a_{i}$ are set equal, $a_{i}=a$. As discussed in [2], the Kerr-AdS metrics in odd spacetime dimensions $D=2 n+1$ can then be written as

$$
\begin{aligned}
d s^{2}= & -\frac{1+r^{2} l^{-2}}{\Xi} d t^{2}+\frac{U d r^{2}}{V-2 m}+\frac{r^{2}+a^{2}}{\Xi}\left[(d \psi+A)^{2}+d \Sigma_{n-1}^{2}\right] \\
& +\frac{2 m}{U \Xi^{2}}[d t-a(d \psi+A)]^{2}
\end{aligned}
$$

where

$$
U=\left(r^{2}+a^{2}\right)^{n-1}, \quad V=\frac{1}{r^{2}}\left(1+r^{2} l^{-2}\right)\left(r^{2}+a^{2}\right)^{n},
$$

$\Xi \equiv 1-a^{2} l^{-2}$, and $d \Sigma_{n-1}^{2}$ is the standard Fubini-Study metric on $\mathbb{C P}^{n-1}$, with Kähler form $J=\frac{1}{2} d A$. The coordinate $\psi$ has period $2 \pi$, and the terms $\left[(d \psi+A)^{2}+d \Sigma^{2}\right]$ in the metric are nothing but the round metric on the unit sphere $S^{2 n-1}$. Following the procedure described above, and noting that in this special case the origin of the AdS metric occurs at $r^{2}=-a^{2}$, we find that the action is given by

$$
\begin{aligned}
I & =\frac{\beta \pi^{n-1}}{4(n-1) ! \Xi^{n} l^{2}} \lim _{R \rightarrow \infty}\left[\left(R^{2}+a^{2}\right)^{n}-\left(r_{+}^{2}+a^{2}\right)^{n}-\left(1-m l^{2} R^{-2 n}\right)\left(R^{2}+a^{2}\right)^{n}\right], \\
& =\frac{\beta \pi^{n-1}}{4(n-1) ! \Xi^{n}}\left[m-l^{-2}\left(r_{+}^{2}+a^{2}\right)^{n}\right] .
\end{aligned}
$$

It is easily seen that this agrees with (A.15), under the specialisation $a_{i}=a$.

When all the rotation parameters are set equal in the Kerr-AdS metric in an even dimension $D=2 n$, then, as discussed in [2], the metric can be written as

$$
\begin{aligned}
d s^{2}= & -\frac{\Delta_{\theta}\left(1+r^{2} l^{-2}\right)}{\Xi} d t^{2}+\frac{U d r^{2}}{V-2 m}+\frac{\rho^{2} d \theta^{2}}{\Delta_{\theta}}+\frac{r^{2}+a^{2}}{\Xi} \sin ^{2} \theta\left[(d \psi+A)^{2}+d \Sigma_{n-2}^{2}\right] \\
& +\frac{2 m}{U \Xi^{2}}\left[\Delta_{\theta} d t-a \sin ^{2} \theta(d \psi+A)\right]^{2}
\end{aligned}
$$

where

$$
\begin{array}{rlrl}
U & =\frac{\rho^{2}\left(r^{2}+a^{2}\right)^{n-2}}{r}, & V=\frac{1}{r}\left(1+r^{2} l^{-2}\right)\left(r^{2}+a^{2}\right)^{n-1}, \\
\Delta_{\theta}=1-a^{2} l^{-2} \cos ^{2} \theta, & \rho^{2}=r^{2}+a^{2} \cos ^{2} \theta, \quad \Xi=1-a^{2} l^{-2},
\end{array}
$$


and $d \Sigma_{n-2}$ is the standard Fubini-Study metric on $\mathbb{C P}^{n-2}$, with Kähler form $J=\frac{1}{2} d A$. Applying the procedure described above for calculating the action, we find in this case

$$
\begin{aligned}
I= & \frac{n !(2 n-1) 2^{2 n-3} \beta \pi^{n-2}}{(2 n) ! \Xi^{n-1} l^{2}} \lim _{R \rightarrow \infty}\left[R\left(R^{2}+a^{2}\right)^{n-1}-r_{+}\left(r_{+}+a^{2}\right)^{n-1}\right. \\
= & \left.\quad-\left(1-m l^{2} R^{1-2 n}\right) R\left(R^{2}+a^{2}\right)^{n-1}\right], \\
(2 n) ! \Xi^{n-1} & \left.\frac{n !(2 n-1) 2^{2 n-3} \beta \pi^{n-2}}{2}-r_{+} l^{-2}\left(r_{+}^{2}+a^{2}\right)^{n-1}\right] .
\end{aligned}
$$

This indeed agrees with (A.16), specialised to the case where $a_{i}=a$.

\section{References}

[1] S.W. Hawking, C.J. Hunter and M.M. Taylor-Robinson, Rotation and the AdS/CFT correspondence, Phys. Rev. D59, 064005 (1999), hep-th/9811056.

[2] G.W. Gibbons, H. Lü, D.N. Page and C.N. Pope, The general Kerr-de Sitter metrics in all dimensions, J. Geom. Phys. 53, 49 (2005), hep-th/0402008.

[3] M. Cvetič, H. Lü and C.N. Pope, Charged Kerr-de Sitter black holes in five dimensions, Phys. Lett. B598, 273 (2004), hep-th/0406196.

[4] M. Cvetič, H. Lü and C.N. Pope, Charged rotating black holes in five dimensional $U(1)^{3}$ gauged $N=2$ supergravity, Phys. Rev. D70, 081502 (2004), hep-th/0407058.

[5] A.S. Eddington, The nature of the physical world, Cambridge University Press 1928.

[6] D. Christodoulou, Phys. Rev. Lett. 25, 1596 (1970).

[7] D. Christodoulou and R. Ruffini, Phys. Rev. D, 2552 (1971).

[8] G.W. Gibbons and S.W. Hawking, Action integrals and partition functions in quantum gravity, Phys. Rev. D15, 2752 (1977).

[9] A. Magnon, On Komar integrals in asymptotically de Sitter spacetimes, J. Math. Phys. 26, 3112 (1985).

[10] B. Carter, Hamilton-Jacobi and Schrödinger separable solutions of Einstein's equations, Commun. Math. Phys. 10, 280 (1968).

[11] M. Henneaux and C. Teitelboim, Asymptotically anti-de Sitter spaces, Comm. Math. Phys. 98, 391 (1985). 
[12] L.F. Abbott and S. Deser, Stability of gravity with a cosmological constant, Nucl. Phys. B195, 76 (1982).

[13] A. Ashtekar and A. Magnon, Asymptotically anti-de Sitter space-times, Class Quant Grav 1, L39 (1984).

[14] V.A. Kostelecky and M.J. Perry, Solitonic black holes in gauged $N=2$ supergravity, Phys. Lett. B371, 191 (1996), hep-th/9512222.

[15] M.M. Caldarelli, G. Cognola and D. Klemm, Thermodynamics of Kerr-Newman-AdS black holes and conformal field theories, Class. Quant. Grav. 17, 399 (2000), hepth/9908022.

[16] J.D. Brown and J.W. York, Quasilocal energy and conserved charges derived from the gravitational action, Phys. Rev. D47, 1407 (1993).

[17] S. Silva, Black hole entropy and thermodynamics from symmetries, Class. Quant. Grav. 19, 3947 (2002), hep-th/0204179.

[18] D. A. Rasheed, Non-linear electrodynamics: Zeroth and first laws of black hole mechanics, hep-th/9702087.

[19] D. Berman and M.K. Parikh, Holography and rotating AdS black holes, Phys. Lett. B463, 168 (1999), hep-th/9907003

[20] S.W. Hawking and H. Reall, Charged and rotating AdS black holes and their CFT duals, Phys. Rev. D61, 024014 (2000), hep-th/9908109.

[21] A.M. Awad and C.V. Johnson, Higher dimensional Kerr-AdS black holes and the AdS/CFT correspondence, Phys. Rev. D63, 124023 (2001), hep-th/0008211

[22] V. Balasubramanian and P. Kraus, A stress tensor for anti-de Sitter gravity, Commun. Math. Phys. 208, 413 (1999), hep-th/9902121.

[23] R.C. Tolman, On the weight of heat and thermal equalibrium in general relativity, Phys. Rev. 35, 904 (1930).

[24] K. Skenderis, Asymptotically anti-de Sitter spacetimes and their stress energy tensor, Int. J. Mod. Phys. A16, 740 (2001), hep-th/0010138.

[25] A. Ashtekar and S. Das, Asymptotically anti-de Sitter space-times: Conserved quantities, Class. Quant. Grav. 17, L17 (2000), hep-th/9911230. 
[26] A. Batrachenko, J.T. Liu, R. McNees, W.A. Sabra and W.Y. Wen, Black hole mass and Hamilton-Jacobi counterterms, JHEP 0505, 034 (2005), hep-th/0408205.

[27] S. Das and R.B. Mann, Conserved quantities in Kerr-anti-de Sitter spacetimes in various dimensions, JHEP 0008, 033 (2000), hep-th/0008028.

[28] K. Landsteiner and E. Lopez, The thermodynamic potentials of Kerr-AdS black holes and their CFT duals, JHEP 9912, 020 (1999), hep-th/9911124.

[29] N. Deruelle and J. Katz, On the mass of a Kerr-anti-de Sitter spacetime in D dimensions, Class. Quant. Grav. 22, 421 (2005), gr-qc/0410135.

[30] J. Katz, A note on Komar's anomalous factor, Class. Quantum Grav. 2, 423, (1985); J. Katz, J. Bičák and D. Lynden-Bell, Relativistic conservation laws and integral constraints for large cosmological perturbations, Phys. Rev. D55, 5759 (1997). 